\title{
AÇÃO AFIRMATIVA NA EDUCAÇÃO SUPERIOR: O CASO DE KERALA NA ÍNDIA
}

\author{
Loni Bordoloi PaZich ${ }^{* *}$
}

RESUMO: Um debate duradouro lançado por Pierre Bourdieu e JeanClaude Passeron em The Inheritors: French Students and Their Relation to Culture (1964) é se as forças que mantém a hierarquia social e sua concomitante distribuição de vantagens e desvantagens podem ser quebradas de forma genuína, significativa e duradoura. Por meio do caso do Estado de Kerala na India, o presente artigo considera o papel das forças históricas na mediaçáo do impacto de uma política de ação afirmativa na educação superior a nível nacional. Devido a uma combinação de fatores, incluindo seu legado de matrilinidade e a experiência com o comunismo, Kerala expandiu, de maneira bem-sucedida, o acesso à educação superior para alunos de castas baixas, incluindo mulheres, em uma proporção que ainda não foi concretizada no restante da Índia.

Palavras-chave: Ação afirmativa; Índia; Casta; Mobilidade social.

\section{AFFIRMATIVE ACTION IN HIGHER EDUCATION: THE CASE OF KERALA IN INDIA}

ABSTRACT: An enduring debate launched by Pierre Bourdieu and JeanClaude Passeron in The Inheritors: French Students and Their Relation to Culture (1964) is whether the forces that maintain social hierarchy and its attendant distribution of advantages and disadvantages can be disrupted in a genuine, meaningful, and lasting manner. Using the case of the Indian state of Kerala, this paper considers the role of historical forces in mediating the impact of a nation-wide affirmative action policy in higher education. Due to a combination of factors, including its legacy of matriliny and experience with communism, Kerala has successfully expanded access to higher education for low-caste students, including women, to an extent that remains unrealized in the rest of India.

Keywords: Affirmative action; India; Caste; Social mobility.

\footnotetext{
* Traduzido por Karin Quast.

** Program Director at the Teagle Foundation, New York City, NY., USA. E-mail de contato: bordoloi@teagle.org
} 


\title{
ACTION POSITIVE DANS L'ENSEIGNEMENT SUPÉRIEUR: LE CAS DU KERALA EN INDE
}

\begin{abstract}
RÉSUMÉ: Un long débat lancé par Pierre Bourdieu et Jean-Claude Passeron dans Les Héritiers: les étudiants et la culture (1964) les forces qui maintiennent la hiérarchie sociale et sa distribution concomitante des avantages et des inconvénients peuvent être réparties d'une manière authentique, significative et durable. À travers le cas de l'Etat du Kerala en Inde, cet article examine le rôle des forces historiques dans la médiation de l'impact d'une politique d'action positive dans l'enseignement supérieur à l'échelle nationale. Grâce à une combinaison de facteurs, y compris son héritage de matrilinéarité et de l'expérience avec le communisme, le Kerala a élargi, d'une manière très efficace, l'accès à l'enseignement supérieur pour les étudiants de caste inférieure, y compris les femmes, une proportion qui n'a pas encore été trouvée nulle part en Inde.
\end{abstract}

Mots-clés: L’Action positive; Inde; Caste; La Mobilité sociale.

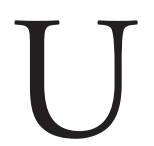

m debate duradouro iniciado por Pierre Bourdieu e Jean-Claude Passeron, em The Inheritors: French Students and Their Relation to Culture (1964), é se as forças que mantêm a hierarquia social e sua concomitante distribuição de vantagens e desvantagens podem ser quebradas de forma genuína, significativa e duradoura. Adotando um continuum de sistemas de reprodução social, desde aqueles que permitem um grau significativo de mobilidade social àqueles que absolutamente não o permitem, as sociedades caracterizadas por sistemas de casta estão no ponto extremo em termos de reprodução automática de grupos sociais fixos geração após geração, com “[...] todas as vantagens e benefícios, exclusões e restrições que, tomadas como um todo, determinam as relações entre as classes dominantes e as classes dominadas [...]". (PASSERON, 1986, p. 621) Grupos de castas são grupos hereditários fechados que determinam a ocupação, posição social, moradia e direitos legais. (LASKAR, 2010) A principal distinção entre o status de casta e o status de classe é que o primeiro é tanto hereditário quanto inflexível; por exemplo, ao passo que alguns grupos de castas baixas tiveram sucesso em ascender seu status economicamente, seu status de casta (e o acesso relativo a parceiros de casamento, por exemplo) permanece inalterado. Passeron (1986, p. 626) observa que um "[...] modelo de reprodução não pode ser também definido como um modelo para a mudança histórica [...]”. Mas, ainda assim, a importância do contexto histórico em romper com sistemas de reprodução, que trazem vantagens a determinados grupos à custa de outros, é inescapável, como pode ser visto no caso excepcional do Estado de Kerala, na Índia.

O Estado de Kerala, no sudoeste da Índia, obteve sucesso em aumentar o acesso à educação superior para alunos provenientes de castas baixas, incluin- 
do as mulheres, em uma proporção que permanece inatingível no restante do país. Ao contrário de alguns estados na Índia, a proporção de gênero sexual em Kerala favorece as mulheres e é a mais alta no país: 1.084 mulheres de todas as procedências para cada 1.000 homens e de 1.057 de castas catalogadas para cada 1.000 homens $^{1}$ (Scheduled castes). (OFFICE OF THE REGISTRAR \& CENSUS COMMISSIONER, 2013b) O censo mais recente realizado na Índia, em 2011, indica que o nível de letramento das mulheres em Kerala em muito ultrapassa as tendências nacionais e é o mais alto no país. (OFFICE OF THE REGISTRAR \& CENSUS COMMISSIONER, 2013a) Kerala também é atípica devido a ter alcançado graus de letramento notavelmente altos para indivíduos provenientes de castas catalogadas. Kerala conseguiu obter tais ganhos apesar de ter uma história muito severa com as divisóes baseadas em castas, e de ser um dos estados menos prósperos na Índia, tomando por base o seu produto interno bruto. Este artigo examina o quanto Kerala conseguiu reduzir a disparidade em relação à igualdade de acesso e sucesso na educação superior para alunos de castas baixas, particularmente as mulheres, e considera como o papel das forças históricas bem como das escolhas de políticas contemporâneas, têm promovido o progresso.

Este artigo se inicia trazendo uma breve descriçáo do antigo sistema de castas da Índia e sua relevância no que tange ao acesso à educação superior contemporânea. Em seguida, traça a história da ação afirmativa na Índia, que teve início no século XIX, sob o Raj Britânico, sua inclusão na constituição da Índia, ratificada em 1950 e sua expansão nos anos 1990. Depois faz uma transição, discutindo o contexto histórico, social e político único de Kerala e que influencia o status das mulheres e seu acesso à educação na atualidade, incluindo sua história de educação básica apoiada pelas antigas famílias reais e sua experiência com costumes matrilineares que permitiram que meninas de alguns grupos de castas tivessem acesso à educação. A seguir, traz uma breve discussão de como a ação afirmativa tem sido implementada em Kerala e os resultados atuais na educação superior vivenciados por alunos de castas catalogadas, particularmente as mulheres, no sistema de educação superior de Kerala. Os avanços alcançados por meios dos esforços históricos no passado interagem com a moderna política de ação afirmativa, de forma a produzir os distintos padróes de participação na educação superior que atualmente vemos para os grupos de castas baixas em Kerala e, em especial, para o sexo feminino.

\section{Castas e a lógica do sistema de reservas}

O sistema de castas tem sido referido como o apartheid oculto da Índia. (VISVANATHAN, 2001) Tradicionalmente, a sociedade Hindu é rigidamente estratificada em vários grupos ou classes distintas: os Brahmins - Brâmanes (sa- 
cerdotes, eruditos e professores); os Kshatriyas - Xátrias (governantes, dignitários e militares); os Vaishyas - Vaixás (comerciantes, negociantes e artesãos), e os Shudras - Sudras (provedores de serviços, agricultores e trabalhadores, operários). (CHAUHAN, 2008) Uma quinta categoria de pessoas não possui casta; são excluídos, tidos como páreas, a escória, forçados a se engajar em tarefas ritualmente poluidoras (impuras), tais como trabalho com couro ou limpeza de esgoto. Cada uma dessas castas possui centenas de subcastas com base em diferenças ocupacionais, regionais, linguísticas e sectárias.

Os indivíduos sem casta agora se autodenominam "Dalits". Os Dalits eram historicamente considerados como "intocáveis", significando que o contato físico com eles ou com algo que possuíssem era considerado impuro. Essa intocabilidade se manifestava, por exemplo, com os Dalits sendo forçados a usar poços diferentes, que não eram confiáveis, ou que eram distantes dos povoados. (HUMAN RIGHTS WATCH, 1999) Em algumas partes da Índia, incluindo Kerala, os Dalits eram inclusive considerados como "não podendo ser vistos" ( "unseeable") e precisavam tomar o cuidado de permanecer a uma distância prescrita dos grupos de castas mais altas para evitar repercussóes. (JEFFREY, 1978; MENON, 1994) Embora atualmente tanto a "intocabilidade" quanto "não poderem ser vistos" seja considerado contra a lei, as injustiças cruéis ainda persistem. Por exemplo, "[...] assassinatos motivados por questôes de casta, estupros e outros abusos são ocorrência diária na Índia [...].”. (HUMAN RIGHTS WATCH, 2012)

As castas catalogadas (Scheduled Casts) ${ }^{2}$ são uma parte significativa da população nacional, com 16,8\%. (OFFICE OF THE REGISTRAR GENERAL \& CENSUS COMMISSIONER, 2013b) Devido ao fato de diferentes grupos de castas terem historicamente podido se engajar em uma faixa estreita e restrita de ocupaçôes, e o status de casta mediar o acesso à educação, a mobilidade social intergeracional dos Dalits permanece extremamente limitada. (MAJUMDER, 2013) O crescimento da populaçáo de castas catalogadas entre 2001 e 2011 foi maior do que aquela vista para a população em geral, com um aumento de $20,8 \%$ versus $17,7 \%$ para a população em geral. (OFFICE OF THE REGISTRAR GENERAL \& CENSUS COMMISSIONER, 2013b) Esse crescimento pode refletir parcialmente novas adiçóes à lista de castas catalogadas: o número de castas catalogadas vem aumentando regularmente desde 1950 e agora inclui 1.241 grupos, contra 1.221 verificados no último censo de 2001. (OFFICE OF THE REGISTRAR GENERAL \& CENSUS COMMISSIONER, 2013b) Em Kerala, cerca de 50 grupos estão listados como castas catalogadas. (KERALA PUBLIC SERVICE COMMISSION, 2003)

A política de ação afirmativa para a educação superior na Índia é a mais ampla e de mais longa duração deste tipo no mundo. (DESHPANDE; YADAV, 2006) Tal política tem a forma de cotas ou "vagas reservadas" na educação superior, direcionadas a alunos provenientes de castas baixas; como resultado, na Índia tal 
política é denominada de "sistema de reserva". As vagas reservadas são separadas para estudantes Dalit cujas afiliaçóes de casta estejam enumeradas em uma "schedule" ou lista de grupos protegidos na constituição indiana. Como consequência, os Dalits também são denominados de "castas catalogadas", termo que usarei predominantemente no restante deste artigo. Aumentar a mobilidade social das castas catalogadas por meio de caminhos tais como o acesso à educação superior não é somente uma questão de justiça compensatória, mas também uma questão de crescimento econômico nacional dado que quase um em cada cinco indianos tem sido historicamente privado de acesso. A atenção aos resultados vivenciados pelas mulheres de tais origens é especialmente importante porque pesquisas indicam que as injustiças de gênero impedem o crescimento econômico na Ásia do Sul. (SAHNI; KALYAN SHANKAR, 2012)

\section{Panorama histórico do sistema de reserva na Índia}

Atualmente, os dispositivos constitucionais que permitiram as reservas para os grupos catalogados foram ampliados para instituições de educação superior com financiamento central (ou seja, federal), proporcionalmente à sua representação em nível nacional (mas não superior a 50\%), ao passo que os estados definem sua própria distribuição de vagas em instituições por eles financiadas de forma a refletir a sua representação aproximada de grupos catalogados. (WEISSKOPF, 2004) As reservas agora se estendem tanto a alunos quanto a professores (tendo em vista que a maioria das instituições de ensino superior são financiadas pelo estado ou pelo governo federal e, portanto, são fontes públicas de emprego). Mesmo os alunos que buscam vagas reservadas devem atender a uma pontuação mínima em exames de admissão competitivos. A estratégia típica de admissão é relaxar as pontuaçóes de entrada por um valor especificado para os grupos catalogados.

A experiência da Índia com o sistema de cotas é anterior à independência em 1947. Em Kerala, a demanda por reservas foi realizada já em 1891, em Travancore, contra o recrutamento de servos civis não nativos, tipicamente os brâmanes Tamil, na engrenagem do governo. (KRISHNAKUMAR, 2004; LASKAR, 2010) A primeira implementação formal dessa política foi registrada em 1902, no estado principesco de Kolhapur na Índia Central, com 50\% de cotas em cargos na administração pública para as "classes atrasadas" ("backward classes") ou desfavorecidas. As reservas foram subsequentemente adotadas tanto no governo colonial britânico como em governos regionais. Por exemplo, a presidência Madras em 1921 e o estado principesco de Travancore em 1936, introduziram cotas na administração pública para os não Brâmanes, os Muçulmanos e alguns grupos Cristãos. (KRISHNAKUMAR, 2004; LASKAR, 2010) 
Após a Índia ter conseguido a independência da Grā-Bretanha em 1947, o ativista Dalit, B. R. Ambedkar foi nomeado como o presidente do comitê do projeto da constituição indiana. (LASKAR, 2010) No início dos anos 1940, Ambedkar havia estabelecido a Federação de Todas as Classes Oprimidas de Toda a Índia (All India Depressed Classes Federation), advogando por reservas no sistema público de governo. Ambedkar foi instrumental em assegurar que a constituição provesse a igualdade de oportunidades a todos os cidadãos e proibisse a discriminação com base em religião, grupo racial, casta, sexo ou local de nascimento, incluindo também a prática da 'intocabilidade'. Curiosamente, a constituiçấo, conforme originalmente ratificada em 1950, incluía provisóes específicas para reservas de emprego na administração pública e na assembleia, mas não na educação, para indivíduos classificados como pertencentes a castas catalogadas ou tribos catalogadas (esses sendo os grupos indianos aborígenes). (LASKAR, 2010) A constituição foi originalmente ratificada com a seguinte cláusula, conhecida como o artigo 46, com relação à educação:

[...] o Estado deve promover com especial cuidado os interesses educacionais e econômicos das seçôes mais fracas da sociedade (em particular, das castas catalogadas e das tribos catalogadas), e deve protegê-los da injustiça social e de todas as formas de exploração [...]. (LASKAR, 2010)

Foram feitas provisóes constitucionais de forma que o Presidente e o Parlamento determinassem quais grupos seriam incluídos no "Schedule" da Constituição (ou lista), ou quais castas e tribos protegidas seriam consideradas elegíveis para as reservas. Essas listas têm sido atualizadas de forma contínua desde a sua introdução, baseadas, em parte, nos dados de censos decenais.

A cláusula que possibilita as reservas na educação foi incluída em 1951, como a primeira emenda constitucional aprovada pelo Parlamento, em resposta a um caso da Suprema Corte, Champakam Dorairajan vs. o Estado de Madras. Neste caso, o pleiteante contesta a prática do estado de reservar vagas nos cursos de medicina e engenharia em faculdades estaduais alegando violar a igualdade de oportunidade para todos os cidadáos sob o Artigo 15 da Constituiçáo, ao passo que o estado sustentava que sua prática estava protegida pelo artigo 46. A Suprema Corte deu ganho de causa ao pleiteante. O Parlamento entáo reagiu, alterando o Artigo 15 e instituindo que:

[...] nada neste artigo [...] deve impedir que o estado faça qualquer provisáo especial para o avanço de qualquer classe de cidadãos social ou educacionalmente atrasada ou para as castas e tribos catalogadas [...]. (LASKAR, 2010) 
Um caso na Suprema Corte em 1963, Balaji vs. o Estado de Mysore, estabeleceu que as reservas para as castas e tribos catalogadas não deveriam exceder $50 \%$ das vagas disponíveis nas faculdades e universidades. (GUPTA, 2006; LASKAR, 2010)

As tentativas de expandir as reservas àqueles pertencentes às "outras classes atrasadas" ("other backward classes"- OBCs) foram lançadas pouco depois das provisóes especiais para as castas e tribos catalogadas terem sido introduzidas na Constituição. (LASKAR, 2010; MOSES, 2010) O Governo central nomeou comissóes em 1953 e 1978, lideradas por K.S. Kalekar e B.P. Mandal respectivamente, para investigar se as reservas deveriam ser estendidas a grupos não-Dalit ou grupos indígenas que, sob alguma circunstância, estivessem em desvantagem. (LASKAR, 2010) Ambas as comissóes recomendaram uma expansão do sistema de reservas para as OBCs. As recomendações da Comissão Mandal foram implementadas cerca de uma década depois pelo Governo central, em 1990, mediante violentos protestos, e ainda permanecem politicamente controversas. A expansão das reservas às OBCs foi mantida pela Suprema Corte em 1993, no caso Indra Sawhney vs. Union of India. A Corte tentou distinguir entre as OBCs genuinamente empobrecidas e aquelas que tinham assegurado um relativo avanço social e econômico, indicando que a "creamy layer" (nata) das OBCs não poderia se beneficiar das reservas. O conceito de creamy layer foi aplicado aos filhos de membros do alto nível de governo e de oficiais militares e aqueles cujos pais possuíssem uma renda superior a 100.000 rúpias (o teto foi subsequentemente elevado para 250.000 rúpias em 2004). (GUPTA, 2006; LASKAR, 2010)

O panorama das reservas continuou a ser alterado ao longo da última década. As reservas em instituições privadas de educação superior, independentemente do nível de financiamento público que recebiam, foi ampliado em 2005 devido a aprovação da 93a . emenda pelo Parlamento. (GUPTA, 2006; LASKAR, 2010) O Ato Central das Instituiçóes Educacionais (Central Educational Institutions Act) aprovado pelo Parlamento em 2006, atualmente aloca vagas em todas as instituições financiadas pelo Governo Central da seguinte forma: $15 \%$ para castas catalogadas; $7,5 \%$ para tribos catalogadas; e $27 \%$ para outras classes desfavorecidas (as backward classes). A validade da 93a . emenda e do Ato Central foram alvo de litígio em 2008, no caso Ashok Kumar Thakur vs. Union of India, mas ambos foram mantidos pela Suprema Corte.

As cotas para mulheres na educação superior estão em vigor em algumas instituições estaduais (INDIAN EXPRESS, 2010; SAHNI \& KALYAN SHANKAR, 2012; SHINDE GOLE, 2011), mas não necessariamente aquelas com fundos do governo central, embora essa situação possa se alterar no futuro. O Projeto de Lei para Reservas para Mulheres foi introduzido pelo Parlamento em 2008, de forma a reservar 33\% das vagas em legislaturas nacionais e estaduais. O projeto foi aprovado pela Câmara Alta (senado) do Parlamento nacional em 
2010; há agora a pressão para que a Câmara Baixa aprove o projeto para que este possa ser sancionado por lei, embora a probabilidade de sua aprovação permaneça incerta. (TIWARI, 2013)

O sistema de reservas continua sendo forte alvo de litígio. Por exemplo, alguns estados, incluindo Kerala, estão alocando vagas para membros pobres das castas “forward" (isto é, altas); a legalidade deste esforço político está perante a Suprema Corte da Índia. (ECONOMIC TIMES, 2010) Além disso, alguns estados excederam a cláusula da Suprema Corte que estabelece que o número de vagas reservadas não ultrapasse metade das vagas e, portanto, estão sob litígio. O litígio também se estende a cotas relativas à nomeação de docentes. Mais recentemente, a Suprema Corte decretou que embora cotas para a docência superior devam ser disponibilizadas para aqueles de origem de castas catalogadas, este requisito não se aplicava a áreas altamente especializadas tais como a Medicina. (MISHRA, 2013)

\section{O contexto histórico, social e político de Kerala}

Kerala está localizada na costa sudoeste da Índia e constitui uma única região linguística onde se fala a língua Malaiala. (JEFFREY, 1978; SEN, 1997) $\mathrm{O}$ estado moderno de Kerala foi criado combinando-se a região de Malabar com os estados principescos de Travancore e Cochin. (JEFFREY, 1987) Os britânicos governavam Malabar diretamente como parte da presidência de Madras e governavam Travancore e Cochin, indiretamente, por meio de conselheiros políticos. Kerala é o $12^{\circ}$ maior estado em termos populacionais na Índia, com 33 milhóes de habitantes. (OFFICE OF THE REGISTRAR GENERAL \& CENSUS COMMISSIONER, 2013C) A região de Kerala possui uma cultura diferenciada por ter se desenvolvido em relativo isolamento: está separada do restante da península indiana pela cadeia de montanhas conhecida como Western Ghats. (JEFFREY, 1978) Além disso, sua longa costa fez nascer uma forte tradição marítima e de comércio, resultando em comunidades de tamanho considerável de Hindus, Muçulmanos e Cristãos, não comparável a qualquer outro local na Índia. Embora Kerala seja um estado majoritariamente Hindu, totalizando $54 \%$ de sua população, $25 \%$ são Muçulmanos e 16\% são Cristãos. (DECCAN CHRONICLE, 2013) As castas catalogadas representam 9;1\% da população do estado. (OFFICE OF THE REGISTRAR GENERAL \& CENSUS COMMISSIONER, 2013b)

Kerala tem sido celebrada há muito tempo por seus altos índices de letramento. (MEYER; BRYSAC, 2011) Kerala lidera em termos de letramento feminino desde a virada do século XX. Como exemplo podemos citar os índices de letramento feminino em Cochin, Malabar e Travancore, que naquela época eram de $4,5 \%, 3 \%$ e $3 \%$ respectivamente, ao passo que o índice nacional era de 0,7\%. (JEFFREY, 1989) Atualmente, seu índice de letramento se equipara ao 
do mundo desenvolvido. Como pode ser visto na Tabela 1, atualmente o índice de letramento feminino em Kerala é de $92 \%$, ultrapassando em muito o índice de letramento feminino nacional, que é de $65 \%$, e sendo o mais alto do país. (OFFICE OF THE REGISTRAR GENERAL \& CENSUS COMMISSIONER, 2013a) O índice de letramento feminino em Kerala permanece forte mesmo nas áreas rurais, em 91\%, comparado à média nacional, que é de 58\%. Embora os índices de letramento para as mulheres de Kerala ainda fiquem atrás daqueles vistos para o sexo masculino, em âmbito nacional a disparidade entre mulheres e homens é muito maior. Por exemplo, os índices de letramento entre mulheres e homens difere em 4 pontos percentuais em Kerala, ao passo que, em âmbito nacional, difere em 16 pontos percentuais.

\section{Tabela 1}

Índices de letramento para homens e mulheres na Índia e Kerala, a populaçáo total e a população rural, 2011

\begin{tabular}{ccc}
\hline \multicolumn{3}{c}{$\begin{array}{c}\text { Indices de letramento para homens e } \\
\text { mulheres na Índia e Kerala }\end{array}$} \\
\hline & India & Kerala \\
\hline População total & $72,99 \%$ & $94,00 \%$ \\
População masculina & $80,89 \%$ & $96,11 \%$ \\
População feminina & $64,64 \%$ & $92,07 \%$ \\
População rural & $67,77 \%$ & $92,98 \%$ \\
População masculina & $77,15 \%$ & $95,53 \%$ \\
População feminina & $57,93 \%$ & $90,81 \%$ \\
\hline
\end{tabular}

Fonte: Office of the Registrar General \& Census Commissioner (2013b)

Kerala atingiu altos índices de letramento para aqueles oriundos de castas catalogadas. Embora o índice de letramento para a população de castas catalogadas seja menor do que o índice da população geral do estado, ela é muito maior do que o índice da população em geral na Índia, como podemos ver na Tabela 2. Por exemplo, o índice de letramento para o sexo feminino de castas catalogadas em áreas rurais, em $81,6 \%$, está atrás daquela para o sexo feminino de todas as origens em áreas rurais, em 90,8\%, mas está muito à frente do índice nacional para mulheres de castas catalogadas, que é de $56.5 \%$. 


\section{Tabela 2}

Índices de letramento para homens e mulheres na Índia e Kerala para indivíduos de todas as origens e para aqueles de castas catalogadas

\begin{tabular}{ccc}
\hline & $\begin{array}{c}\text { Todas as } \\
\text { origens }\end{array}$ & $\begin{array}{c}\text { De origem de castas } \\
\text { catalogadas }\end{array}$ \\
\hline $\begin{array}{c}\text { Índia } \\
\text { População }\end{array}$ & $73,00 \%$ & $66,10 \%$ \\
masculina & $80,90 \%$ & $75,20 \%$ \\
$\begin{array}{c}\text { População } \\
\text { feminina }\end{array}$ & $64,60 \%$ & $56,50 \%$ \\
$\begin{array}{c}\text { Kerala (somente } \\
\text { Rural) }\end{array}$ & $92,98 \%$ & $87,60 \%$ \\
$\begin{array}{c}\text { População } \\
\text { masculina }\end{array}$ & $95,35 \%$ & $93,80 \%$ \\
$\begin{array}{c}\text { População } \\
\text { feminina }\end{array}$ & $90,81 \%$ & $81,60 \%$ \\
\hline
\end{tabular}

Fonte: National Institute of Rural Development, N.D.; Office of the Registrar General \& Census Commissioner, (2013b)

Observaçáo: Os dados para a Índia, com relação a todas as origens e de castas catalogadas, são de 2011. Os dados acerca de letramento em Kerala são apenas para a área rural. Os dados relativos a indivíduos de todas as origens para Kerala são de 2011, ao passo que para as castas catalogadas em áreas rurais os dados săo de 2009-2010.

Uma série de fatores sociais, culturais e históricos exclusivos de Kerala contribui para o status das mulheres, incluindo seus altos níveis de letramento que, por sua vez, posicionaram este estado de forma a colher maiores resultados da política de ação afirmativa na contemporaneidade. Tais fatores incluem seus costumes sociais matrilineares, o financiamento da educação desde os antigos governantes principescos, e os esforços realizados por missionários cristãos. (JEFFREY, 1978; ROSS, 2006) Além desses fatores, a história política de Kerala com o comunismo pode ter contribuído para explicar porque a população pertencente às castas catalogadas está em uma situação melhor se comparada a seus semelhantes em âmbito nacional, ao menos em termos de indicadores de desenvolvimento humano, como o letramento.

\section{Sistema matrilinear no grupo de casta Nair}

As mulheres de um grande grupo de castas em Kerala, conhecido como os Nairs (alternativamente soletrado como Nayyar), historicamente gozam de relativa autonomia devido a práticas matrilineares segundo as quais a origem 
dos descendentes e da propriedade é estabelecida a partir da mãe. Embora os Nairs não sejam nem Brâmanes nem Xátrias, eles constituem, no entanto, um grupo de casta "respeitável" de proprietários de terras e administradores públicos. (JEFFREY, 1989, p. 19) As práticas matrilineares dos Nairs foram firmemente estabelecidas em torno do século XVI e floresceram durante o século XIX, porém desapareceram por completo em torno da metade do século XX. (JEFFREY, 1987; 2004) No "tipo ideal" de família Nair, a propriedade passava da mãe para a filha mais velha, mas era gerenciada pelo membro masculino mais velho para o benefício de toda a família. (JEFFREY, 2004) As mulheres da casa recebiam parceiros sexuais Brâmanes, Xátrias ou Nair, que elas podiam livremente escolher ou descartar. Os filhos resultantes eram considerados Nair e eram criados pelas mães. As mulheres Nair que se casassem tinham maior autonomia para sair de seus casamentos e tipicamente não deixavam a casa da família; ao contrário, elas eram matrilocais, no sentido de que o marido visitava a esposa e seus filhos. (JEFFREY, 1987) Embora as mulheres Nair não exercessem controle econômico direto sobre os bens herdados, elas eram encorajadas a receber educação e tinham a liberdade de falar com homens e de aparecer em público.

Quando, no início dos anos 1870, oportunidades de emprego que demandam letramento emergiram para as mulheres em Kerala, tais como parteiras e professoras, os benefícios da educação das mulheres ganharam uma utilidade óbvia ainda maior. (JEFFREY, 1987, 1989) As famílias de castas baixas aspiravam seguir o exemplo definido pelos Nair, que serviam de "grupo de referência para a sociedade Kerala” (JEFFREY, 2004, p. 132), porque eles gozavam de um nível incomum de mobilidade social, incluindo relaçóes sancionadas com os Brâmanes. Os Nair tornaram socialmente aceitável que as meninas fossem educadas, que tivessem liberdade de movimento em público e que frequentassem locais como escolas, onde entravam em contato com garotos. (JEFFREY, 1989) Jeffrey (2004, p. 658) resume o impacto das práticas sociais e culturais dos Nairs em padróes mais amplos de participação educacional, conforme segue:

[...] a matrilinidade criou condiçóes nas quais algumas garotas de alto status, em algumas partes de Kerala, aprenderam a ler e a escrever, tiveram mais liberdade de movimento do que a maioria das mulheres de alto status de seu tempo em qualquer outro lugar na Índia, e algumas dessas mulheres foram trabalhar em empregos assalariados. As mulheres de outras castas e religiôes também o fizeram, porque se isso era aceitável para os Nairs, líderes sociais da antiga Kerala, então era desejável para a maioria das outras mulheres [...]. 


\section{A tradição histórica do financiamento público da educação}

Kerala "[...] teve a boa sorte de ter famílias reais em Travancore e Cochin que eram atipicamente a favor da educação básica [...].”. (SEN, 1997, p. 16) A difundida educação vernácula teve início no século XIX devido aos esforços das famílias reais governantes. (MEYER \& BRYSAC, 2011; ROSS, 2006; SEN, 1997) Por exemplo, um decreto emitido pela rainha Laxmi Parvathi Bai de Travancore, em 1817, determina o seguinte:

[...] o estado deveria financiar todo o custo da educação de seu povo a fim de que não haja atrasos na expansão do esclarecimento entre ele, para que por meio da difusão da educação eles possam se tornar sujeitos e servidores públicos melhores e que a reputação do estado possa deste modo ser melhorada [...]. (ROSS, 2006, p. 173)

Até os anos 1870 o governo de Travancore havia estabelecido um diretor de educação vernácula, um comitê de livros vernáculos incumbido de escrever e traduzir livros didáticos, bem como subvenções com base na matrícula em escolas de ensino elementar que atendessem determinados padróes. (JEFFREY, 1987)

Os costumes sociais e os incentivos a favor de emprego permitiram que ao menos as mulheres pertencentes a alguns grupos de castas continuassem sua educação quando a educação superior de estilo britânico foi introduzida no século XIX. A título de exemplo, Gilbert Salte, um professor de economia na Universidade de Madras no início do século XX, observou que na Faculdade de Maharaja em Travancore (agora a University College em Thiruvananthapuram),

[...] o interesse especial era o de que esta admitia homens e mulheres sem distinção, e a garotas que pareciam totalizar cerca de um quinto do total, compartilhavam de maneira igualitária todas as atividades da faculdade, exceto pelo futebol e jogos semelhantes. Os alunos de ambos os sexos eram quase que exclusivamente das duas castas de Nairs e cristãos indianos [...]. (SARADAMONI, 1994, p. 504)

Embora apenas determinados grupos de castas se beneficiassem da educação que recebia financiamento público, isso tinha um amplo impacto no sentido de que tanto garotos como garotas tinham acesso a ela. 


\section{O impacto dos missionários cristãos}

A comunidade cristã de Kerala abrange três grupos principais: os cristáos sírios que parecem ter sido Brâmanes de alto status e que estabelecem a sua conversão a Tomás, o Apóstolo; católicos de rito latino de comunidades costeiras e de pescadores de baixo status que foram convertidos pelos portugueses no século XVII; e protestantes dos níveis mais baixos da sociedade Hindu, incluindo os $D a-$ lits, que foram convertidos por missionários britânicos no século XIX. (JEFFREY, 1989) A educação feminina entre os cristãos sírios de alto status e os católicos de rito latino de status mais baixo era incomum se comparada aos Nairs. Por exemplo, em 1875, a proporção de mulheres letradas que eram Nair era de 50\% e de mulheres que eram cristãs era de $30 \%$, embora as mulheres Nair e as cristâs abrangessem $20 \%$ cada da população total. (JEFFREY, 1989) A chegada dos missionários protestantes encorajou os esforços para difundir o letramento devido à nova competição, entre as variadas seitas, em efetuar o proselitismo e a conversão. (JEFFREY, 1978) Os missionários também demonstravam a familiar motivação em assegurar que seus novos convertidos, inclusive as mulheres, fossem letrados, a fim de que pudessem melhor participar de seus estudos religiosos e espalhar mensagens cristâs. (ROSS, 2006) Em 1839, por exemplo, o Registro Missionário (Missionary Register) observou que, "[...] todos os cristãos que realmente almejam o avanço do reino de Cristo na Índia admitem que, na prossecução desse objeto, a educação feminina é uma das principais coisas que deve ser promovida [...].". (ROSS, 2006, p. 174)

\section{Os Dalits e o Marxismo}

O comunismo tem um forte legado em Kerala, que se tornou o primeiro estado no mundo a ter um governo comunista, eleito democraticamente em 1957. Menon (1994) afirma que a "[...] maior razão dos comunistas terem chegado ao poder em [Kerala]... foi a transformaçáo do comunismo em uma doutrina de igualdade de castas [...].”. (p. 2) A inclemência do sistema de castas em Kerala, conforme exemplificado pela prática da "invisibilidade", contribuiu para a consciência de classe entre as castas mais baixas. Embora "os sem-terra formassem o núcleo do apoio comunista" (NOSSITER, 1982, p. 337), o movimento por fim teve sucesso porque atraiu as castas mais altas. A emergência e viabilidade política do comunismo coincidiram com o colapso da matrilinearidade, dado que a legislaçấo destruiu os costumes de indivisibilidade da propriedade e herança através da linha feminina, e novos costumes sociais europeus e do norte da Índia romperam com as noçóes predominantes de casamento e sexualidade. (JEFFREY, 1978) O apagamento da matrilinearidade e a perda do apoio econômico e familiar que a 
acompanhavam causaram impacto não apenas nos Nairs, mas também nas outras castas altas que tinham conexóes com eles. Como resultado, houve a emergência de uma geraçáo de jovens homens e mulheres de casta mais alta que náo tinha "[...] nem a propriedade por direito próprio, nem um pai ou tio materno que estivesse pronto a se responsabilizar por eles [...].”. (JEFFREY, 1978, p. 7) Os primeiros líderes comunistas tendiam a ser homens de origem Nair, e seu disseminado letramento facilitou a divulgação das ideias marxistas. (JEFFREY, 1978)

Desde os anos 1950, o empoderamento político das castas mais baixas em Kerala, por meio do movimento comunista, contribuiu para o seu bem-estar em relação a outras partes da Índia. Por exemplo, Kerala tornou-se o primeiro estado na Índia a abolir o feudalismo em 1970, uma prática que virtualmente escravizava os Dalits. Mais recentemente, o Partido Comunista pediu reservas para castas catalogadas junto a empregadores privados, não apenas públicos. (THE HINDU, 2012) Aumentar o nível de letramento encorajou o consumo de jornais - Kerala possui o maior consumo per capta de jornais na Índia (ROSS, 2006) - e participação no discurso político. Desde os anos 1980 Kerala tem sido governada alternadamente pelo Partido Comunista da Índia e pelo Partido do Congresso Nacional, sendo que cada partido precisa formar coalizóes com partidos regionais menores a fim de governar. Isto "[...] estabeleceu um padrão distinto de acomodação, permitindo que as reivindicaçóes de grupos de minoria sejam explicitamente reconhecidas [...].” (KAILASH, 2011, p. 183) e no qual os políticos são forçados a responder às demandas dos eleitores, incluindo os eleitores das castas catalogadas. (JEFFREY, 1978; SEN, 1997) No âmbito da educação, tanto a liderança política como o envolvimento popular encorajaram sua expansão aos grupos de castas mais baixas.

\section{A implementação da Ação afirmativa em Kerala na atualidade}

As cotas variam em instituiçóes financiadas centralmente (ou seja, pelo Governo central) e em instituiçóes financiadas pelos estados, sendo os níveis de cotas para alunos de castas catalogadas definidos de forma a refletir suas proporções em nível nacional (nas instituiçóes financiadas centralmente) e por estado (nas instituições financiadas pelos estados). A distribuição das vagas reservadas é extremamente complexa, pois varia por estado, por instituição e, em alguns casos, por curso universitário. (WEISSKOPF, 2004) Em Kerala, nas instituiçóes financiadas pelo estado, as vagas são normalmente distribuídas da seguinte maneira: $8 \%$ para castas catalogadas; $2 \%$ para tribos catalogadas; e 26\% para classes "atrasadas" social ou educacionalmente (socially or educationally backward classes: SEBC). (GOVERNMENT OF KERALA, 2012b) A categoria SEBC é subdividida de forma que a atribuição de vagas atenda vários grupos, incluindo 
muçulmanos, católicos de rito latino, outros cristãos "atrasados" e outros Hindus "atrasados". Sob este esquema típico de distribuição, 36\% das vagas são reservadas nas instituiçóes estaduais de Kerala. (Lembrem-se de que nas instituiçóes financiadas centralmente, a atribuição é a seguinte: 15\% para castas catalogadas; $7,5 \%$ para tribos catalogadas; e $27 \%$ para outras classes "atrasadas"). De acordo com os regulamentos de Kerala, as pontuaçóes de corte para candidatos de castas catalogadas são normalmente relaxadas em 5\%. (RAJAN, 2011) Há algumas exceçôes à norma estadual de atribuição de vagas, por exemplo, a Universidade de Calcutá - no nordeste de Kerala - reserva 50\% de suas vagas, incluindo 15\% para candidatos provenientes de castas catalogadas e $10 \%$ para alunos de castas "avançadas" (forward) que estejam abaixo da linha de pobreza. (UNIVERSITY OF CALICUT, 2013) De forma similar, os programas de Mestrado em Ciências da Computação possuem diferentes atribuições de vagas reservadas, dependendo dos alunos estarem tentando se matricular em faculdades estaduais (cujo percentual é de $46 \%$ e inclui membros pobres das castas "avançadas") ou em faculdades privadas (cujo percentual é de 36\%). (GOVERNMENT OF KERALA, 2012a) Este artigo passa agora às consideraçóes acerca dos resultados vivenciados pelos alunos de castas catalogadas, especialmente do sexo feminino, na educação superior de Kerala.

\section{Tendências relativas ao acesso e sucesso na educação superior em Kerala}

Os dados mais recentes disponíveis acerca dos índices de participação, na educação superior, desagregados por status de casta e gênero, são coletados via a Pesquisa de Educação Superior de toda a Índia, conduzida pelo Ministério do Desenvolvimento de Recursos Humanos do governo indiano. A pesquisa foi lançada em 2001 e é ministrada a todas as universidades reconhecidas, faculdades afiliadas e institutos de educação técnica, de enfermagem e formação de professores. Da mesma forma que em todas as pesquisas, os dados são influenciados pelo índice de respostas e a qualidade das informaçóes fornecidas pelos respondentes, neste caso, as instituições. A taxa de resposta variou por tipo de instituição, com participação de $100 \%$ das universidades de Kerala (em comparação a $89 \%$ de todas as universidades em nível nacional) e com a participação de 58\% e 63\%, respectivamente, de faculdades e institutos em Kerala (comparados a 50\% e 51\%, respectivamente, com a taxa de participação de toda a Índia). (MINISTRY OF HUMAN RESOURCE DEVELOPMENT, 2013, p. A-19) O Ministério calcula a taxa bruta de matrículas (TBM) como a proporção de alunos matriculados na educação superior referente ao grupo de 18-23 anos de idade. Calcula o índice de paridade de gênero como a proporção da TBM do sexo feminino para a TBM do 
sexo masculino para um dado nível educacional. Os cálculos de alguns autores são relatados a seguir, com base nos valores disponibilizados pelo Ministério a partir da pesquisa. Os dados relacionados, especificamente às mulheres de castas catalogadas, são limitados, assim como o são os dados sobre resultados, tais como a obtenção do certificado de bacharelado.

Atualmente Kerala possui 962 instituiçóes de educaçáo superior, sendo 29 instituiçóes para cada 100.000 alunos e uma média de matrícula, por instituição de 557 alunos. (MINISTRY OF HUMAN RESOURCE DEVELOPMENT, 2013, p. T-4) No país, há 23 instituiçóes para cada 100.000 alunos, com uma média de 700 alunos matriculados por instituiçáo. Essas tendências podem ser o resultado parcial da alta densidade populacional de Kerala, que atualmente é de 860 pessoas por $\mathrm{km} 2$, contra 368 pessoas por $\mathrm{km}^{2}$ no âmbito nacional (OFFICE OF THE REGISTRAR GENERAL \& CENSUS COMMISSIONER, 2013c) e seu sucesso em assegurar que suas instalaçôes educacionais estejam distribuídas equilibradamente pelo estado. (ROSS, 2006) Em todo o estado, para alunos de todas as procedências, a distribuição de gênero nas matrículas na educação superior é de $57 \%$ para o sexo feminino e $43 \%$ para o sexo masculino. (MINISTRY OF HUMAN RESOURCE DEVELOPMENT, 2013, p. 18) O padrão em Kerala, onde as mulheres representam a maioria das matrículas na educação superior, deriva da norma vista nacionalmente, onde a divisão de gênero é de $55 \%$ para o sexo masculino e de $45 \%$ para o sexo feminino. Na verdade, as mulheres possuem uma representaçáo desproporcionalmente maior na educação superior em Kerala devido a sua parcela no grupo de 18-23 anos, calculada em 49,9\%. (cálculos do autor; MINISTRY OF HUMAN RESOURCE DEVELOPMENT, 2013, p. T-68) As meninas de Kerala fazem a transiçâo de cada estágio do processo educacional de forma bem sucedida, passando pelo que no Brasil é denominado de educação fundamental I e II e ensino médio, estando, portanto, posicionadas de forma a participar da educação superior. (HUSAIN; SARKAR, 2010)

Conforme pode ser verificado na Tabela 3 , as TBMs na educaçáo superior para o grupo de 18-23 anos, a partir de 2010-2011 são muito favoráveis às mulheres de Kerala, incluindo aquelas provenientes de castas catalogadas, em relação às tendências vistas em nível nacional. Em Kerala, a TBM do estado para todos os alunos e para aqueles de castas catalogadas excedeu os padróes vistos no âmbito nacional. As TBMs das mulheres excederam a dos homens para todos os alunos $(25,1$ vs. 18,8$)$ e para alunos de castas catalogadas $(18,1$ vs. 10,3). Em Kerala, a TBM para todo o estado para todos os alunos e para aqueles de castas catalogadas excedeu os padróes vistos nacionalmente. As TBMs para as mulheres excederam as dos homens para todos os alunos $(25,1$ vs. 18,8) e para os alunos de castas catalogadas $(18,1$ vs. 10,3). Em nível nacional a tendência se reverteu: as TBMs para os homens excederam as TBMs para as mulheres para todos os alunos $(20,8$ vs. 17,9$)$ e para alunos de castas catalogadas $(14,6$ vs. 12,3$)$. O progresso 
que Kerala fez em matricular alunos provenientes de castas catalogadas é rápido e recente. As TBMs para alunos de castas catalogadas foram 2,3, 8,1 e 16,7 em 1993-1994, 1999-2000 e 2004-2005, respectivamente (GEORGE, 2011), comparadas a 14,2 em 2010-2011.

\section{Tabela 3}

Taxas brutas de matrícula para todos os alunos e alunos de castas catalogadas por gênero na Índia e em Kerala, 2010-2011

\begin{tabular}{ccc}
\hline & $\begin{array}{c}\text { Todas as } \\
\text { procedências }\end{array}$ & $\begin{array}{c}\text { Procedência de } \\
\text { castas catalogadas }\end{array}$ \\
\hline Índia & 19,4 & 13,5 \\
Sexo masculino & 20,8 & 14,6 \\
Sexo feminino & 17,9 & 12,3 \\
Kerala & 21,9 & 14,2 \\
Sexo masculino & 18,8 & 10,3 \\
Sexo feminino & 25,1 & 18,1 \\
\hline
\end{tabular}

Fonte: Ministry of Human Resource Development (2013b, p. 25, T-56)

Os índices de paridade de gênero na educação superior para o grupo de 18-23 anos de idade também exibem tendências positivas na participação feminina de castas catalogadas. $O$ índice de paridade de gênero é de 1,76 para alunas de castas catalogadas contra 1,34 para alunas de todas as procedências em Kerala, comparado a 0,84 para alunas de castas catalogadas e 0,86 para alunas de todas as procedências em nível nacional. (MINISTRY OF HUMAN RESOURCE DEVELOPMENT, 2013b, p. T-57)

Embora esses indicadores sobre a participação das mulheres e de castas catalogadas na educação superior sejam positivos, eles camuflam os tipos de faculdade/programa e as oportunidades de mobilidade ocupacional. Os dados disponíveis sugerem que, embora Kerala tenha obtido sucesso em fornecer acesso às mulheres e às castas catalogadas, esse acesso tende a se concentrar em programas na área de Artes e Ciências, que são cursos de menor prestígio e tipicamente não possuem fortes perspectivas de emprego no mercado de trabalho indiano. (GEORGE, 2011; WEISSKOPF, 2004) De todos os alunos de castas catalogadas matriculados na educação superior em Kerala em 2008, mais de 80\% estavam matriculados em faculdades de Artes e Ciências, ao passo que as matrículas em faculdades de engenharia e medicina, que são consideradas como tendo mais prestígio e valor no mercado de trabalho indiano, pouco excediam 10\%. (cálculos do autor; GEORGE, 2011) Além disso, os alunos de castas catalogadas represen- 
tavam $14,5 \%$ e 7,9\%, respectivamente, do total de matrículas em faculdades de artes e ciências e faculdades politécnicas. (GEORGE, 2011) Em outras palavras, os alunos de castas catalogadas estão sobre-representados nas faculdades de artes e ciências e sub-representados em faculdades politécnicas, que são as que conduzem a empregos de melhor remuneração. A participação por gênero também é distorcida. De todas as mulheres matriculadas na educação superior em Kerala em 2004, 92\% estava na área de artes e ciências, enquanto somente 3,7\% e 4,2\% estavam em cursos de engenharia e medicina, respectivamente. (SAHNI \& KALYAN SHANKAR, 2012)

Os dados sobre a conclusão do curso universitário são escassos e refletem questóes de capacidade mais ampla com coleta regular de dados, avaliação e relatórios. (MATHEWS, 2010) Para os alunos de castas catalogadas que conseguem se matricular em faculdades de engenharia, a pesquisa sugere que embora eles não tendam a abandonar o curso, eles gastam tempo significativo para se graduar, pois não passam nos exames. (RAJAN, 2011) A título de exemplo, para aqueles matriculados em uma pequena amostragem de faculdades, quase três quartos dos alunos de castas catalogadas não passaram na sua primeira tentativa em seus exames no oitavo (e que deveria ter sido o último) semestre do curso de engenharia, comparados a um quinto quando se trata de alunos de castas não catalogadas.

De maneira geral, a pesquisa existente sugere que Kerala tem obtido sucesso em assegurar o acesso à educação superior para as castas catalogadas, incluindo as mulheres, e aumentou a mobilidade ocupacional para esses grupos ao longo do tempo. (MAJUMDER, 2013) É importante lembrar que as cruéis desigualdades baseadas em castas permanecem no estado. Por exemplo, a proporção de pessoas de origem de castas catalogadas que vive na pobreza nas áreas urbanas de Kerala é de 32,5\%, contra 7,8\% para aquelas que não são provenientes de castas catalogadas. (GEORGE, 2011) Ainda assim, o progresso que Kerala conseguiu atingir ao abrir a educação superior a alunos provenientes de castas baixas, incluindo as mulheres, é notável.

O sistema de reserva foi desenvolvido para compensar as desigualdades baseadas em castas profundamente consolidadas e avançar em relação à justiça social na sociedade indiana. (MOSES, 2010) Toda a Índia vivenciou a mesma intervenção de políticas como uma forma de expandir a mobilidade social para grupos de castas catalogadas (embora a implementação de tal política, em termos de distribuição de cotas, varie de estado para estado). Ao contrário do restante da Índia, Kerala fez progressos significativos em direção a corrigir as diferenças de equidade muito mais rapidamente para esses grupos: seu contexto histórico e político único, incluindo o valor colocado na educação das mulheres e seu ethos igualitário, devido à influência do comunismo, amplificaram o impacto da política de ação afirmativa. 


\section{Notas}

1. N.T.: Encontramos várias traduçôes em documentos e trabalhos científicos para o termo scheduled castes: castas listadas, castas específicas, castas classificadas, castas catalogadas. Optamos por utilizar o termo "catalogadas".

2. N.T.: As Scheduled Casts - SCs e as Scheduled Tribes - STs (os habitantes originais) são designações oficiais conferidas a vários grupos de pessoas historicamente desprivilegiadas.

\section{Referências}

ALTBACH P. G.; MATHEWS, E. Progressive state. Times Higher Education, 28 oct. 2010. BOURDIEU, P.; PASSERON, J.-C The Inheritors: french students and their relation to culture. [trad. by Richard Nice]. Chicago: The University of Chicago Press, 1979.

CHAUCHAN, C. P. S. Education and caste in India. Asia Pacific Journal of Education, v. 28, n. 3, p. 217-234, 2008.

COLLEGES to have 20\% reservation for girls. Indian Express, 17 Jun 2010.

ENSURE job quota for Dalits in private sector: Prakash Karat. The Hindu, 13 Dec 2012.

GEORGE, K. K. Higher education in Kerala: How inclusive is it to scheduled castes and scheduled tribes? Working Paper n. 1(4). Centre for the Study of Social Exclusion and Inclusive Policy, Cochin University of Science and Technology, 2011.

GOVERNMENT OF KERALA G.O. (Rt.) No: 2445/2012/H.Edn, 2012a.

GOVERNMENT OF KERALA. Prospectus for admission to professional degree courses. 2012b. [G.O.(Ms). no. 355/2011/H.Edn].

GUPTA, A. Affirmative action in higher education in India and the US: A study in contrasts Berkeley, CA: Center for Studies in Higher Education, University of California, Berkeley, 2006.

HUMAN RIGHTS WATCH. Broken people: Caste violence against India's "Untouchables". New York: Human Rights Watch, 1999.

. India: UN Members Should Act to End Caste Discrimination. Human Rights Watch News, 14 May 2012.

HUSAIN, Z.; SARKAR, S. Gender disparities in educational trajectories in India: Do females become more robust at higher levels? Social Indicators Research, v. 101, n. 1, p. 37-56, 2010.

JEFFREY, R. Matriliny, Marxism, and the birth of the communist party in Kerala, 19301940. Journal of Asian Studies, v. 38, n. 1, p. 77-98, 1978.

Governments and culture: How women made Kerala literate. Pacific Affairs, v. 60, n. 3, p. 447-72, 1987. 
JEFFREY, R. Women and the "Kerala" model: Four lives, 1870s. South Asia, v. 12, n. 2, p. 13-32, 1989.

. Legacies of Matriliny: The Place of Women and the "Kerala Model." Pacific Affairs, v. 77, n. 4, p. 647-664, 2004.

KAILASH, K. K. Political outsourcing as a coalition strategy in Kerala. In MAHAJAN G. (Org.). Accommodating diversity: ideas and institutional practice. New Delhi, India: Oxford University Press, 2011.

KERELA heralds population fall. Deccan Chronicle, 12 may 2013.

KERALA PUBLIC SERVICE COMMISSION. List of scheduled castes in the Kerala state. Available at: http://www.keralapsc.org/scstobc.htm. Retrieved on 6 Dec 2013.

KRISHNAKUMAR, R. A history of reservation. Frontline, v. 21, n. 17, 14 Aug. 2004.

LASKAR, M. H. Rethinking Reservation in Higher Education in India. ILI Law Review, v. 1 , n. 1 , p. 25,2010 .

MAJUMDAR, R. Intergenerational mobility: A study of social classes in India. New York: Springer, 2013.

MATHEWS, E. Paucity of data on Indian higher education. Economic and Political Weekly, 2010.

MENON, D. M. Caste, nationalism and communism in south India: Malabar, 1900-1948. Cambridge, UK: Cambridge University Press, 1994.

MEYER, K. E.; BRYSAC, S. B. Kerala: Multiple Improbabilities. World Policy Journal, v. 28, n. 4, p. 60-69, 2011.

MINISTRY OF HUMAN RESOURCE DEVELOPMENT. All India survey on higher education: 2010-11. New Delhi: Government of India, Ministry of Human Resource Development, Department of Higher Education, 2013.

MISHRA, A. Uproar over affirmative action exemption for medical schools. University World News, 15 Aug. 2013.

MOSES, M. S. Moral and Instrumental Rationales for Affirmative Action in Five National Contexts. Educational Researcher, v. 39, n. 3, p. 211-228, 2010.

NATIONAL INSTITUTE OF RURAL DEVELOPMENT. Section-10: Scheduled castes and scheduled tribes: Rural development statistics, 2011-12. Hyderabad, Andhra Pradesh, India. Disponível em: http://www.nird.org.in/Rural\%20Development\%20Statistics\%20 2011-12/data/sec-10.pdf. Acesso em $12 \mathrm{dez} 2013$.

NOSSITER, T. J. Communism in Kerala: A study in political adaptation. Berkeley \& Los Angeles: University of California Press, 1982.

OFFICE OF THE REGISTRAR GENERAL \& CENSUS COMMISSIONER. Executive Summary: Census 2011 Highlights for Kerala. New Delhi, India: Government of India, Ministry of Home Affairs, Office of the Registrar General \& Census Commissio- 
ner. Disponível em: http://www.censusindia.gov.in/2011census/PCA/PCA_Highlights/ pca_highlights_file/kerala/Exeutive_Summary.pdf. Acesso em 12 dez 2013a.

OFFICE OF THE REGISTRAR GENERAL \& CENSUS COMMISSIONER. Census of India 2011: Final population totals: Dashboard. CensusInfo India 2011. Disponível em: http://www.censusindia.gov.in/2011census/censusinfodashboard/index.html. Acesso em 5 dez 2013b.

. Census of India, 2011: Primary Census Abstract: Scheduled Castes and Scheduled Tribes. New Delhi, India. Disponível em: http://censusindia.gov.in. Acesso em $12 \mathrm{dez}$ 2013c.

PASSERON, J. Theories of socio-cultural reproduction. International Social Science Journal, v. 38, n. 4, p. 619-629, 1986.

RAJAN, K. Performance and problems of scheduled caste and scheduled tribe students in the engineering colleges of Kerala. Kerala State Higher Education Council, 2011.

ROSS, K. Status of women in highly literate societies: the case of Kerala and Finland. Literacy, v. 40, n. 3, p. 171-178, 2006.

SAHNI, R.; KALYAN SHANKAR, V. Girls' higher education in India on the road to inclusiveness: on track but heading where? Higher Education, v. 63, n. 2, p. 237-256, 2012.

SARADAMONI, K. Women, Kerala and Some Development Issues. Economic and Political Weekly, v. 29, n. 9, p. 501-509, 1994.

SC notice to Kerala got for reserving seats for forward communities. Economic Times, 12 may 2010.

SEN, A. Radical needs and moderate reforms. In: DREZE, J.; SEN, A. (Org.). Indian development: Selected regional perspectives. Oxford: Oxford University Press, 1997. p. 1-27.

SHINDE GOLE, S. 30\% girls' quota likely in junior college. The Times of India, 10 Dec. 2011.

TIWARI, R. Pawar writes to PM to get women’s Bill passed. Indian Express, 4 Dec. 2013.

UNIVERSITY OF CALICUT. Category of seats and reservation rules, 2013. Disponível em: http://www.uoc.ac.in/ugsw/crprpt/ReservationRules.pdf. Acesso em 12 dez 2013.

VISVANATHAN, S. The race for caste: Prolegomena to the Durban conference. Economic and political weekly, v. 36, n. 27, p. 2512-2516, 2001.

WEISSKOPF, T. E. Impact of Reservation on Admissions to Higher Education in India. Economic and Political Weekly, v. 39, n. 39, p. 4339-4349, 2004.

Recebido em 29 de agosto de 2014.

Aprovado em 18 de março de 2015. 